\title{
EFICÁCIA DO HERBICIDA FLUMIOXAZIN APLICADO ISOLADAMENTE E EM MISTURA COM DIURON OU DICLOSULAM, E DIFERENTES ADJUVANTES, EM ALGODÃO
}

\author{
Eduardo A.D. da Costa ${ }^{1}$, Ednei de C. Macedo ${ }^{1}$, Albino Rozanski ${ }^{1}$ e Marcus B. Matallo ${ }^{1}$ \\ 'Pesquisador Científico. Instituto Biológico, Centro Experimental, Laboratório da Ciência das Plantas Daninhas. Caixa Postal 70. \\ Campinas, SP 13001-970 costaead@biologico.br

\section{RESUMO} \\ O objetivo da presente pesquisa foi avaliar a éficácia e seletividade do herbicida flumioxazin aplicado em pós-emer- \\ gência isoladamente e na mistura em tanque com diuron ou diclosulam, na cultura do algodão. Adotou-se o delineamento de \\ blocos ao acaso, com quatro repetições. Foram estudados os seguintes tratamentos: flumioxazin a $30 \mathrm{~g} / \mathrm{ha}$, flumioxazin+diuron \\ $(25+800$ e $25+1000 \mathrm{~g} / \mathrm{ha})$, flumioxazin+diclosulam $(25+30$ e $25+35 \mathrm{~g} / \mathrm{ha}), \mathrm{MSMA}+$ diuron $(1800+1000 \mathrm{~g} / \mathrm{ha}) \mathrm{como}$ trata- \\ mento padrão, além de duas testemunhas, uma capinada e outra infestada, para as comparações nas avaliações de fitotoxicidade \\ e de controle, respectivamente. Aos herbicidas adicionou-se óleo mineral Assist a 0,5\% v/v ou Triomax a 0,25\% v/v como \\ adjuvantes. O efeito dos herbicidas foi avaliado aos 25 e 39 dias após a aplicação (DAA) e a fitointoxicação aos 10 e 25 DAA. \\ As espécies Commelina benghalensis (trapoeraba), Ipomoea grandifolia (corda-de-viola) e Raphanus raphanistrum (nabiça) \\ foram controladas eficientemente pelo herbicida flumioxazin, isoladamente ou em mistura com diuron ou diclosulam até 39 \\ DAA, com comportamento semelhante ao constatado para o tratamento padrão. Não foram observadas diferenças significa- \\ tivas na produção entre os tratamentos com herbicidas e deles com a testemunha capinada. As plantas de algodão (cv. \\ Coodetec 401) apresentaram leves sintomas de injúrias por ação fitotóxica dos produtos químicos, nos tratamentos envolven- \\ do flumioxazin em mistura com diuron na maior dose e com diclosulam em ambas as doses, porém com rápida e plena \\ recuperação, sem afetar o rendimento da cultura."
}

Palavras-chave: Gossypium hirsutum, mistura em tanque, óleos minerais, seletividade.

\section{ABSTRACT \\ Efficacy of flumioxazin applied alone and in a tank mix with diuron or diclosulam added with different adjuvants in cotton}

A field experiment was carried out in Itapira, SP, to evaluate the selectivity and efficiency of the herbicide flumioxazin alone or in a tank mix with diuron or diclosulam applied in post-emergence to weed control in cotton cv. Coodetec 401 , with two different mineral oils. The experimental design was a randomized block with four replications and the following treatments: flumioxazin (30 g/ha), flumioxazin+diuron $(25+800$ and $25+1,000 \mathrm{~g} / \mathrm{ha})$, flumioxazin+diclosulam $(25+30 \mathrm{and} 25+35 \mathrm{~g} / \mathrm{ha})$, MSMA+diuron $(1,800+1,000 \mathrm{~g} / \mathrm{ha})$ with standard treatment and controls with and without weeds. The adjuvants used were Assist at $0,5 \% \mathrm{v} / \mathrm{v}$ and Triomax at $0,25 \% \mathrm{v} / \mathrm{v}$. The results showed that flumioxazin herbicide alone or in a tank mix with diuron or diclosulam have controlled efficiently Commelina benghalensis L., Ipomoea grandifolia (Dammer) O'Don. and Raphanus raphanistrum L. until 39 days after the post-emergence application. The two adjuvants had no significant effect on the results. None of the chemical treatments affected the cotton plant development or yield.

Key words: Gossypium hirsutum, herbicide mixture, mineral oils, selectivity. 


\section{INTRODUÇÃO}

A contribuição dos agroquímicos para a economia agrícola mundial tem sido crescente e entre eles destacam-se os herbicidas (Chow et al., 1989). Contudo, para que o ingrediente ativo de um agroquímico possa expressar sua fitotoxicidade, é necessário que seja adequadamente formulado pois, isoladamente o mesmo apresenta menos de $10 \%$ de sua atividade biológica potencial (Van Valkenburg, 1982). Dessa forma, diferentes produtos químicos são adicionados ao ingrediente ativo de modo a permitir não só sua solubilização e dispersão como também para aumentar a deposição, espalhamento, molhamento, adesão, retenção, absorção e translocação (Van Valkenburg, 1982; Chow et al., 1989; Foy, 1992; Hochberg, 1996; Holloway, 1998; Zabkiewicz, 2000). Tem ocorrido um crescimento de mercado para os adjuvantes, dentre estes os óleos minerais emulsionáveis, que aumentam o espalhamento das gotas pulverizadas nas superfícies das folhas, incrementando a absorção dos herbicidas aplicados em pós-emergência sobre as plantas alvo (Hess, 1997).

O objetivo do presente trabalho foi avaliar, em condições de campo, o efeito da adição de dois diferentes óleos minerais ao herbicida flumioxazin aplicado isoladamente e em mistura com diuron ou diclosulam, em pós-emergência tardia das plantas daninhas, na cultura do algodão cv. Coodetec 401.

\section{MATERIAL E MÉTODOS}

No ano agrícola 1999/00 foi conduzido um experimento em área localizada no município de Itapira, SP. O solo foi classificado como de textura argilosa, com 50,3\% de argila, $13,3 \%$ de areia grossa, $11,7 \%$ de areia fina e $24,5 \%$ de limo. As análises químicas revelaram pH (água): 5,9; $\mathrm{P}$ (resina): $50,0 \mathrm{mg} / \mathrm{dm}^{3} ; \mathrm{K}^{+}: 0,7 \mathrm{mmol} / \mathrm{dm}^{3} ; \mathrm{Ca}^{+2}: 4,6 \mathrm{mmol} /$ $\mathrm{dm}^{3} ; \mathrm{Mg}^{+2}: 1,2 \mathrm{mmol} / \mathrm{dm}^{3} ; \mathrm{Al}^{+3}$ : não detectado; $\mathrm{H}^{+}: 4,2$ mmol $/ \mathrm{dm}^{3}$; CTC: $10,7 \mathrm{mmol} / \mathrm{m}^{3} ; \mathrm{V}(\%): 60,7$ e M.O.: 21,0 $\mathrm{g} / \mathrm{dm}^{3}$. O preparo do solo constou de uma aração e três gradagens. $\mathrm{O}$ cultivar de algodão utilizado foi Coodetec 401, sendo a semeadura realizada em 14/12/99, com espaçamento de $0,76 \mathrm{~m}$, utilizando-se 15 sementes por metro de sulco. A adubação de semeadura foi de $350 \mathrm{~kg} /$ ha da fórmula 04:14:08. $\mathrm{O}$ delineamento experimental adotado foi o de blocos ao acaso, com quatro repetições. As parcelas mediam 2,4 m x 5,0 $\mathrm{m}$, perfazendo $12 \mathrm{~m}^{2}$. Considerou-se como área útil as duas linhas centrais.

Os herbicidas (Tabela 1) foram aplicados em jato dirigido às entrelinhas no dia 14/02/2000, em pós-emergência da cultura e das plantas daninhas, nos seguintes estádios de desenvolvimento: trapoeraba (Commelina benghalensis), 4 a 8 folhas; corda-de-viola (Ipomoea grandifolia), 2 a 6 folhas; nabiça (Raphanus raphanistrum), 6 a 8 folhas e o algodão em R1 (primeiro botão floral). Ipomoea grandifolia, com uma população média de 8 plantas por $\mathrm{m}^{2}$, foi avaliada no

Tabela 1. Tratamentos com herbicidas e suas respectivas doses. Itapira, SP, 1999/2000.

\begin{tabular}{|c|c|c|c|c|}
\hline Nome comum & $\begin{array}{l}\text { Dose } \\
\text { (g/ha) }\end{array}$ & $\begin{array}{l}\text { Nome } \\
\text { comercial }\end{array}$ & $\begin{array}{l}\text { Formulação } \\
\text { e concentração }\end{array}$ & Adjuvante \\
\hline Flumioxazin & 30 & Flumyzin & $\mathrm{PM}^{1}$ & Assist $(0,5 \% \mathrm{v} / \mathrm{v})$ \\
\hline Flumioxazin + diuron & $25+800$ & Flumyzin+Kamex & $\mathrm{PM}^{1}$ e $\mathrm{PM}^{2}$ & Assist $(0,5 \% \mathrm{v} / \mathrm{v})$ \\
\hline Flumioxazin+diuron & $25+1000$ & Flumyzin+Kamex & $\mathrm{PM}^{1}$ e $\mathrm{PM}^{2}$ & Assist $(0,5 \% \mathrm{v} / \mathrm{v})$ \\
\hline Flumioxazin+diclosulam & $25+30$ & Flumyzin+Spider & $\mathrm{PM}^{1}$ e $\mathrm{GRDA}^{3}$ & Assist $(0,5 \% \mathrm{v} / \mathrm{v})$ \\
\hline Flumioxazin+diclosulam & $25+35$ & Flumyzin + Spider & $\mathrm{PM}^{1}$ e GRDA ${ }^{3}$ & Assist $(0,5 \% \mathrm{v} / \mathrm{v})$ \\
\hline MSMA+diuron & $1800+1.000$ & MSMA+ Kamex & $\mathrm{CS}^{4}$ e $\mathrm{PM}^{2}$ & Assist $(0,5 \% \mathrm{v} / \mathrm{v})$ \\
\hline Flumioxazin & 30 & Flumyzin & $\mathrm{PM}^{1}$ & Triomax $(0,25 \% \mathrm{v} / \mathrm{v})$ \\
\hline Flumioxazin+diuron & $25+800$ & Flumyzin+Kamex & $\mathrm{PM}^{1}$ e $\mathrm{PM}^{2}$ & Triomax $(0,25 \% \mathrm{v} / \mathrm{v})$ \\
\hline Flumioxazin+diuron & $25+1000$ & Flumyzin+Kamex & $\mathrm{PM}^{1}$ e $\mathrm{PM}^{2}$ & Triomax $(0,25 \% \mathrm{v} / \mathrm{v})$ \\
\hline Flumioxazin+diclosulam & $25+30$ & Flumyzin+Spider & $\mathrm{PM}^{1}$ e $\mathrm{GRDA}^{3}$ & Triomax $(0,25 \% \mathrm{v} / \mathrm{v})$ \\
\hline Flumioxazin+diclosulam & $25+35$ & Flumyzin + Spider & $\mathrm{PM}^{1}$ e GRDA ${ }^{3}$ & Triomax $(0,25 \% \mathrm{v} / \mathrm{v})$ \\
\hline MSMA+diuron & $1800+1000$ & MSMA+ Kamex & $\mathrm{CS}^{4}$ e $\mathrm{PM}^{2}$ & Triomax $(0,25 \% \mathrm{v} / \mathrm{v})$ \\
\hline
\end{tabular}

${ }^{1}$ Pó molhavel contendo $500 \mathrm{~g} / \mathrm{L}$ de ingrediente ativo (i.a.).

${ }^{2}$ Pó molhavel contendo $800 \mathrm{~g} / \mathrm{L}$ de i.a.

${ }^{3}$ Grânulos autodispersíveis em água contendo $840 \mathrm{~g} / \mathrm{kg}$ de i.a.

${ }^{4}$ Concentrado solúvel contendo $720 \mathrm{~g} / \mathrm{L}$ de i.a. 
experimento pelos danos que pode causar na qualidade da fibra, na colheita mecânica e no beneficiamento. Utilizou-se um pulverizador costal, a pressão constante (mantida por $\mathrm{CO}_{2}$ comprimido) de $2,11 \mathrm{kgf} / \mathrm{cm}^{2}$, munido de barra com quatro pontas de pulverização XR80.02, na velocidade de $3,6 \mathrm{~km} / \mathrm{h}$ e consumo equivalente a $200 \mathrm{~L} / \mathrm{h}$ a de calda. No momento da aplicação, realizada entre 9 e 11 horas, a temperatura do ar era de $28,4^{\circ} \mathrm{C}$, umidade relativa do ar de $63 \%$ e velocidade do vento de $3,9 \mathrm{~km} / \mathrm{h}$. Os tratamentos fitossanitários constaram de aplicações comerciais de Decis $25 \mathrm{CE}+$ Thiodan CE (0,4+1,5 L/ha) e Azodrim 400 (0,6 L/ha) nos estádios iniciais da cultura. Nos estádios intermediários e finais, foram incluidas aplicações de Thiovit Sandoz (5,0 kg/ha), Folidol 600+Decis 25 CE $(0,8+1,5 \mathrm{~L} / \mathrm{ha})$ e Thiodam CE $(1,5 \mathrm{~L} / \mathrm{ha})$. Aproximadamente 80 dias após o plantio procedeu-se a aplicação do regulador de crescimento PIX a $0,5 \mathrm{~L} / \mathrm{ha}$. Para dessecação das folhas do algodoeiro foi utilizado Dropp na dose de 100 a $150 \mathrm{~g} / \mathrm{ha}$. A colheita foi realizada manualmente, no dia 09/05/00, sendo os dados de produção submetidos à análise de variância pelo teste $\mathrm{F}$, com as médias comparadas pelo teste de Tukey, ambos ao nível de 5\% de probabilidade.

O efeito dos tratamentos sobre a cultura e as plantas daninhas foi avaliado de forma direta aos 25 e 39 dias após a aplicação (DAA) através da contagem da população das espécies, em cada parcela, numa área de $1,0 \mathrm{~m}^{2}$. Os dados coletados foram transformados em percentagem em relação à infestação existente nas parcelas antes da aplicação dos tra- tamentos, e considerada como $100 \%$. A partir destes dados conceituou-se o desempenho dos tratamentos, estabelecendo-se como índice mínimo o controle de $80 \%$ da população da planta daninha (IMC-80). A seletividade e a ação fitotóxica sobre a cultura foram avaliadas respectivamente pela produção e por observações visuais da sintomatologia de injúria aos 10 e 25 DAA, utilizando-se a escala de notas da ALAM (1974) modificada, onde a nota 1 representa nenhum efeito e 5 destruição completa da cultura.

\section{RESULTADOS E DISCUSSÃO}

O herbicida flumioxazin, aplicado isoladamente na dose de $30 \mathrm{~g} / \mathrm{ha}$, com adição do óleo mineral Assist a 0,5\% v/ $\mathrm{v}$, apresentou aos $39 \mathrm{DAA}$ índices de controle superiores ao IMC-80, atingindo $100 \%$ para C. benghalensis, $90 \%$ para $I$. grandifolia e $96 \%$ para $R$. raphanistrum. Com a adição de Triomax $0,25 \% \mathrm{v} / \mathrm{v}$, os índices também foram superiores ao IMC-80 para todos os tratamentos com herbicidas (Tabela 2). Os resultados obtidos para a espécie $C$. benghalensis foram superiores àqueles encontrados por Constantin et al.(2000), quando utilizaram tratamentos similares, porém sem a adição de adjuvantes.

Nas misturas de flumioxazin com diuron nas doses de $25+800$ e $25+1000 \mathrm{~g} / \mathrm{ha}$, os índices de controle das espécies oscilaram entre 96 e 100\%, tanto com a adição do óleo mineral Assist a 0,5\% v/v quanto de Triomax a $0,25 \%$ v/v. Resul-

Tabela 2. Efeito da adição dos adjuvantes aos tratamentos com herbicidas no controle de plantas daninhas e sua seletividade à cultura de algodão cv. Coodetec 401. Os dados são médias de quatro repetições. Itapira, SP, 1999/2000.

\begin{tabular}{|c|c|c|c|c|c|c|c|c|c|c|}
\hline \multirow{2}{*}{ Tratamento } & \multirow{2}{*}{$\begin{array}{l}\text { Dose } \\
\text { (g/ha) }\end{array}$} & \multicolumn{6}{|c|}{ Controle $(\%)$} & \multicolumn{2}{|c|}{$\begin{array}{l}\text { Injúria } \\
\text { (DAA) }\end{array}$} & \multirow{2}{*}{$\begin{array}{c}\text { Produção } \\
\text { (g/tratamento) }\end{array}$} \\
\hline & & 25 & 39 & $\frac{\text { as ap }}{25}$ & $\frac{1108}{39}$ & 25 & 39 & & & \\
\hline & & \multicolumn{2}{|c|}{ C. benghalensis } & \multicolumn{2}{|c|}{ I. grandifolia } & \multicolumn{2}{|c|}{ R. rophonistrum } & & & \\
\hline Flumioxazin 1 & 30 & 100 & 100 & 100 & 90 & 100 & 96 & 1 & 1 & $1773,8 \mathrm{a}$ \\
\hline Flumioxazin+diuron ${ }^{1}$ & $25+800$ & 100 & 98 & 100 & 100 & 100 & 97 & 1 & 1 & $1775,0 \mathrm{a}$ \\
\hline Flumioxazin+diuron ${ }^{1}$ & $25+1000$ & 98 & 100 & 100 & 100 & 99 & 99 & 2 & 1 & $1735,0 \mathrm{a}$ \\
\hline Flumioxazin+diclosulam ${ }^{1}$ & $25+30$ & 100 & 100 & 100 & 100 & 100 & 99 & 2 & 1 & $1706,3 \mathrm{a}$ \\
\hline Flumioxazin+diclosulam ${ }^{1}$ & $25+35$ & 100 & 100 & 100 & 100 & 100 & 100 & 2 & 1 & $1768,8 \mathrm{a}$ \\
\hline MSMA+diuron ${ }^{1}$ & $1800+1000$ & 100 & 100 & 100 & 100 & 100 & 96 & 1 & 1 & $1802,5 \mathrm{a}$ \\
\hline Flumioxazin ${ }^{2}$ & 30 & 100 & 100 & 100 & 100 & 100 & 99 & 1 & 1 & $1831,3 \mathrm{a}$ \\
\hline Flumioxazin+diuron ${ }^{2}$ & $25+800$ & 100 & 100 & 100 & 100 & 100 & 100 & 1 & 1 & $1843,8 \mathrm{a}$ \\
\hline Flumioxazin+diuron ${ }^{2}$ & $25+1000$ & 100 & 99 & 100 & 100 & 100 & 96 & 2 & 1 & $1817,5 \mathrm{a}$ \\
\hline Flumioxazin+diclosulam² & $25+30$ & 100 & 99 & 100 & 100 & 100 & 94 & 2 & 1 & $1802,5 \mathrm{a}$ \\
\hline Flumioxazin+diclosulam & $25+35$ & 100 & 100 & 100 & 100 & 100 & 100 & 2 & 1 & $1906,3 \mathrm{a}$ \\
\hline $\mathrm{MSMA}+$ diuron $^{2}$ & $1800+1000$ & 100 & 100 & 100 & 100 & 100 & 91 & 1 & 1 & $1828,8 \mathrm{a}$ \\
\hline Testemunha capinada & - & 100 & 100 & 100 & 100 & 100 & 100 & 1 & 1 & $1896,3 \mathrm{a}$ \\
\hline Testemunha s/ capina ${ }^{3}$ & - & \multicolumn{2}{|c|}{ (24) } & \multicolumn{2}{|c|}{ (8) } & \multicolumn{2}{|c|}{ (96) } & 1 & 1 & $1310,0 \mathrm{~b}$ \\
\hline $\bar{F}$ & & & & & & & & \multicolumn{3}{|c|}{$3,36^{*}(\mathrm{p}<0,05)$} \\
\hline DMS & & & & & & & & & & 158,66 \\
\hline C.V. $(\%)$ & & & & & & & & & & 8,2 \\
\hline
\end{tabular}

${ }^{1}$ Tratamentos adicionados de Assist a 0,5\% v/v.

${ }^{2}$ Tratamentos adicionados de Triomax a $0,25 \% \mathrm{v} / \mathrm{v}$.

${ }^{3} \mathrm{~N}^{\circ}$ de indivíduos $/ \mathrm{m}^{2}$ (dados médios das parcelas experimentais).. 
tados semelhantes foram obtidos por Carvalho et al. (2000) ao avaliarem a eficiência de herbicidas na cultura de algodão. As misturas de flumioxazin com diclosulam nas doses de $25+30$ e $25+35 \mathrm{~g} / \mathrm{ha}$, com a adição dos adjuvantes, também mostraram índices de controle entre 94 e 100\%, concordando com os resultados obtidos por Benatti et al. (2000) com relação a espécie $C$. benghalensis, ao utilizarem a mistura de flumioxazin em mistura com diclosulam nas doses de $15+35$ e $40+35 \mathrm{~g} / \mathrm{ha}$, em tratamento de pré-emergencia e na dessecação, no manejo da cultura de soja.

Para a mistura MSMA+diuron $(1800+1000 \mathrm{~g} / \mathrm{ha})$, com os adjuvantes Assist e Triomax, não se observaram diferenças no controle das espécies com relação ao IMC-80, em comparação com a eficácia do herbicida flumioxazin aplicado isoladamente ou em mistura com diuron ou diclosulam.

As médias de produção, analisadas pelo teste de Tukey, mostraram-se semelhantes independentemente do adjuvante utilizado, podendo-se concluir que tanto Triomax $(0,25 \% \mathrm{v} /$ v) como o Assist $(0,5 \% \mathrm{v} / \mathrm{v})$ produziram o mesmo efeito no rendimento do algodão em caroço. Dower Neto et al. (2000), testando a eficiência dos óleos minerais Assist 0,5\% v/v e Herbitensil $0,2 \%$ v/v e dos óleos vegetais de algodão, soja e girassol a $0,5 \% \mathrm{v} / \mathrm{v}$, não observaram diferenças significativas nas produções dos tratamentos entre adjuvantes, quando adicionados ao herbicida clethodim na cultura do girassol. Considerando-se a ação fitotóxica dos herbicidas aos 10 DAA, pode ser observado que as plantas de algodão cv. Coodetec 401 mostraram leves sintomas de injúria nos tratamentos envolvendo as misturas de flumioxazin+diuron $(25+1000 \mathrm{~g} /$ ha) e com diclosulam nas doses de 30 e $35 \mathrm{~g} /$ ha para ambos os adjuvantes, sem refletir na produção. Aos 25 DAA as plantas tratadas apresentaram desenvolvimento normal e semelhante à testemunha capinada. Nenhum dos tratamentos ocasionou fitointoxicação, não interferindo na produção. Esses resultados foram semelhantes aos observados por Constantin et al. (2000) e Carvalho et al. (2000), ao estudarem o flumioxazin isoladamente e em mistura com ghyphosate ou sulfosate e diuron e/ou glufosinato na cultura de algodão.

\section{CONCLUSÕES}

O herbicida flumioxazin aplicado isoladamente na dose de $30 \mathrm{~g} /$ ha e em mistura com diuron $(25+800$ e $25+1000$ $\mathrm{g} / \mathrm{ha})$ ou diclosulam $(25+30$ e $25+35 \mathrm{~g} / \mathrm{ha})$ foi eficaz no controle das espécies Commelina benghalensis, Ipomoea grandifolia e Raphanus raphanistrum, independentemente do adjuvante adicionado à calda de aplicação. Não foram observadas diferenças significativas no rendimento do algodão quando aos herbicidas adicionaram-se os adjuvantes Assist $0,5 \% \mathrm{v} / \mathrm{v}$ ou Triomax $0,25 \% \mathrm{v} / \mathrm{v}$. Os tratamentos envolvendo flumioxazin em mistura com diuron na maior dose e com diclosulam em ambas as doses apresentaram leves sintomas de injúrias nas plantas de algodão no início, com posterior recuperação, não afetando o rendimento da cultura.

\section{LITERATURA CITADA}

ASOCIACIÓN LATINOAMERICANA DE MALEZAS (ALAM). Recomendaciones sobre unificación de los sistemas de evaluación en ensayos de control de malezas. ALAM, Bogotá, v.1, n.1, p. 35-38, 1974.

BENATTI, F.V.; ROSSETO, J.; SADE, M. Misturas de diclosulam para controle de plantas daninhas na cultura da soja. In: CONGRESSO BRASILEIRO DA CIÊNCIA DAS PLANTAS DANINHAS, 22. Foz do Iguaçu, PR, 2000. Resumos... Londrina, PR: SBCPD, 2000. p.114.

CARVALHO, F.T.; CESTARE, M.A.; ALVARENGA, S.L.A. Eficiência de herbicidas aplicados em jato dirigido no controle de plantas daninhas na cultura do algodão (Gossypium hirsutum). In: CONGRESSO BRASILEIRO DA CIÊNCIA DAS PLANTAS DANINHAS, 22. Foz do Iguaçu, PR, 2000. Resumos... Londrina, PR: SBCPD, 2000. p.340.

CHOW, P.N.P.; GRANT, C.A.; HINSHALWOOD, A. M.; SIMUNDSSON, E. M.L.S. (Eds.). Adjuvants and agrochemicals: mode of action and physiological activity. Manitoba: CRC Press, 1989. 207p.

CONSTANTIN, J.; OLIVEIRA JR., R.S.; RAMIREZ, A.C.; MARCHIORI JR., O.; MACIEL, C.D.G. Alternativas químicas para a operação de manejo na cultura de algodão. In: CONGRESSO BRASILEIRO DA CIÊNCIA DAS PLANTAS DANINHAS, 22. Foz do Iguaçu, PR, 2000. Resumos... Londrina, PR: SBCPD, 2000. p.330.

DOWER NETO, J.B.; ALVES, C.R. DE L.; CASTILHO, D.; RIBEIRO,F.L.; GOMES, G.B.; SALIM JR, H.M.; GUEDES, M.; PELOSO, S. Avaliação da eficiência e seletividade de herbicidas inibidores de ACCase misturados com diferentes tipos de óleo, na cultura de girassol ( Helianthus annuus L.) In: CONGRESSO BRASILEIRO DA CIÊNCIA DAS PLANTAS DANINHAS, 22, Foz do Iguaçu, PR. Resumos... Londrina, PR: SBCPD, 2000. p.108.

FOY, C.L. Adjuvants: terminology, classification, and mode of action. In: CHOW, P.N.P.; GRANT, C.A.; HINSSHALWOOD, A.M.; SIMUNDSSON, E. (Ed). Adjuvants and agrochemicals: mode of action and physiological activity, Vol II. Boca Raton: CRC Press, 1992. p. 605-626.

HESS, F.D. Adjuvants. In: HERBICIDES ACTION COURSE, 1997, Summary of lectures. West Lafayette: Purdue University, 1997. p. 38-61. 
HOCHBERG, E.G. The market for agricultural pesticide, inert ingredients and adjuvants. In: FOY, C.L.; PRITCHARD, D.W. Pesticide formulation and adjuvant technology. Boca Raton: CRC Press, 1996. p. 203-208.

HOLLOWAY, P.J. Improving agrochemical performance: possible mechanisms for adjuvancy. In: Chemistry and technology of agrochemical formulation. Dordrecht: Kluwer Academic Publishers, 1998. p. 232-63.
VAN VALKENBURG, J.W. Terminology, classification, and chemistry. In: HUGSON, R.F.H. Adjuvants for herbicides. Champaign: Weed. Science Society of America, 1982. p. 1-8

ZABRIEWICZ, J.A. Adjuvants and herbicidal efficacy present status and future prospects. Weed Research., v. 40, n. 1, p. 139-149, 2000. 
